\title{
The U Loop in the Vectorcardiogram with the Frank System
}

Seiichi Toyama, M.D. and Keiko Suzuki, M.D.

$R$ EPORTS of the $U$ wave in the electrocardiogram have been made by I many investigators, even though the significance of the $U$ wave does not seem to have been made clear. Takayasu" has described that the negative $U$ wave appeared mainly in patients with hypertension and coronary sclerosis and the prognosis of such cases were poor. However, the U loop in the vectorcardiogram has not been reported except by Sano and his associates. $\left.{ }^{2}\right)$

It was difficult to confirm the contour of the U loop, because the loop was very small and often outshone by brightness. Recently we have successfully confirmed the U loop in VCG with left ventricular hypertrophy and other abnormal VGG with the Sanborn VGG equipment. Then we have watched carefully and observed the characteristic contour and location of the $U$ loop. Discussions are made in this paper as to the difference between normal and abnormal U loops.

\section{Material and Method}

The U loop in VCG with the Frank system was presently studied in normal people and hypertensive patients. The VCG in hypertensive patients were divided into 4 subgroups; Subgroup I consisted of the cases with a normal range 0 to 69 degree) of the angle of the maximum $\mathrm{T}$ vector in horizontal plane, Subgroup II consisted of the cases in a range of angle between 70 to 109 degree, and Subgroup III referred to those with a range of 110 degree and more. Subgroup IV consisted of the cases with a round or elliptical $T$ loop in horizontal plane. The U loop was studied mainly in horizontal plane and its inscription, location and contour were compared among these groups. The $\mathbf{U}$ loop of patients with aortic insufficiency and anterior and anterolateral infarction were also studied.

\section{RESULTS}

Normal Subjects (Fig. 1)

Horizontal Plane: The $\mathrm{U}$ loop in horizontal plane made a small loop ( $\gamma$ shape) on extension of the T loop. However, it was difficult to differentiate the $\mathrm{U}$ loop from the end of the $\mathrm{T}$ loop. In some cases, the contour of the $\mathrm{U}$

From the Department of Cardiology, Center for Adult Diseases, Osaka.

Received for publication November 11, 1965. 
H.P.

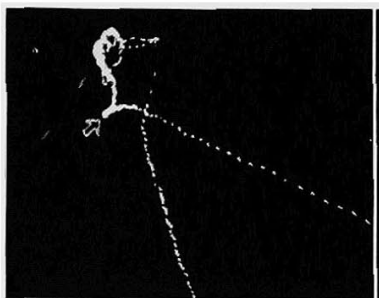

L.S.P.

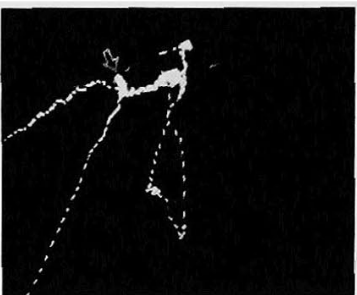

F.P.

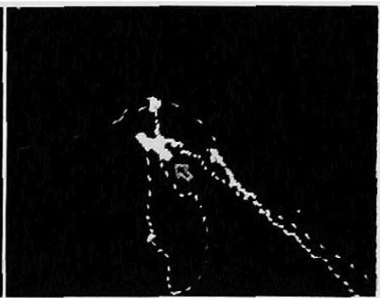

B. Normal U loop in horizontal plane

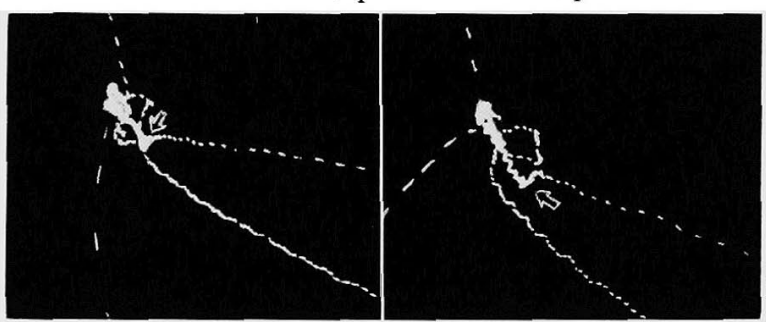

Fig. 1. Normal U loop.

loop was $\mathrm{V}$ shape, and it was hard to recognize the $\mathrm{U}$ loop on the film.

Frontal Plane: The U loop made a clubbing contour or $\gamma$ shape.

Left Sagittal Plane: The U loop was located anteriorly with the small loop directed superiorly in most cases.

Hypertensive Cases (Fig. 2)

Horizontal Plane: The contours of the $U$ loop in these subgroups were various. In cases of Subgroup I, 5 cases belonged to the normal contour of

A. Normal U loop in case with hypertension

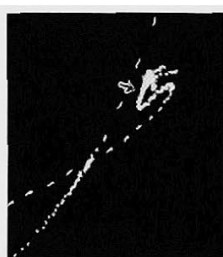

5th type

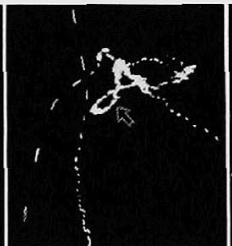

4th type

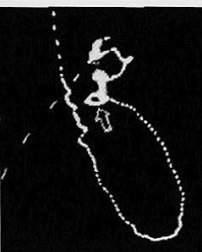

3rd type

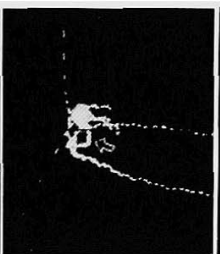

2nd type

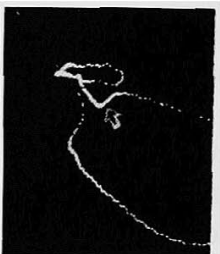

1st type

B. Horizontal U loops in case with aortic insufficiency

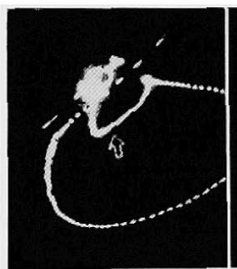

large U loop

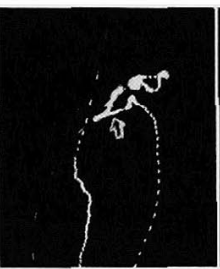

5 th type

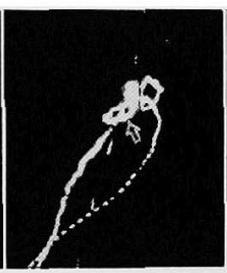

4th type

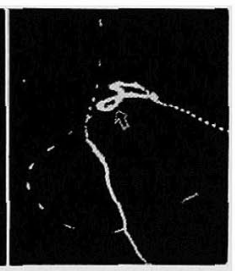

characteristic type

Fig. 2. Secondary U loop change. 
the $\mathrm{U}$ loop (first type), 5 cases to $\mathrm{V}$ shape (second type), 8 cases to $\gamma$ shape located leftanteriorly (second type), one case to $r$ shape located anteriorly (third type) and 3 cases to $\gamma$ shape located rightanteriorly (fourth type). In Subgroup II, 3 cases belonged to $\gamma$ shape located leftanteriorly (second type), 6 cases to third type, 7 cases to fourth type. Only 1 case showed $\mathrm{V}$ shape located rightanteriorly (last or fifth type). In 15 out of 16 cases of Subgroup III, the U loop showed V shape (fifth type). The direction of inscription of this type was clockwise, and the location was rightanteriorly. But 1 case of this group showed the round $\mathrm{U}$ loop with a counterclockwise inscription. In 2 cases of Subgroup I and 4 cases of Subgroup II, the location or contour of the $U$ loop was different from the other cases. Namely, the $\mathrm{U}$ loop located rightposteriorly or rightward with large loop, or the large round or elliptical U loop was situated anteriorly. These U loops were exceptional in Subgroup I-III.

In the abnormal $\mathrm{T}$ loop group (Subgroup IV), 4 cases belonged to second type of the $\mathrm{U}$ loop, 1 case to third type and 9 cases to that located rightanteriorly with $\gamma$ or $\mathrm{V}$ shape (fourth and fifth types). These $\mathrm{U}$ loops were similar to that in above mentioned types of the U loop in Subgroup I-III. In some cases of the remainder, the $\mathrm{U}$ loop showed $\gamma$ or $\mathrm{V}$ shape, but was located rightposteriorly or rightward. In cases of the $U$ loop located rightanteriorly, the $U$ loop was round and large, or $\gamma$ shape or $V$ shape with reverse inscription to the $\gamma$ or V shape in fifth or fourth type of Subgroup I-III. The contour of the $\mathrm{U}$ loop located anteriorly or leftanteriorly was round or elliptical and large (Fig. $3 \mathrm{~A}$ ).

Frontal Plane: In cases of the horizontal $\mathrm{U}$ loop located rightanteriorly (fourth or fifth type), the $U$ loop in frontal plane was located rightward with triangular shape or $r$ shape. However, in cases of the horizontal U loop located leftanteriorly or anteriorly, the U loop in frontal plane showed small loop or club shape and was located leftinferiorly.

When the horizontal $U$ loop of the exceptional shape was located leftanteriorly, the $\mathrm{U}$ loop in frontal plane was located leftinferiorly and was round or elliptical shape.

Sagittal Plane: In sagittal plane, the U loop was located anteroinferiorly or anteriorly and showed a small or large loop with counterclockwise inscription. However, in cases of the horizontal U loop with V shape (fifth type), the sagittal U loop showed V shape.

Aortic Insufficiency Group (Fig. 2 B)

Horizontal Plane: The $\mathrm{U}$ loops in cases with aortic insufficiency were similar to those in hypertensive cases. However, the third type of the U loop was not found in this group. Instead of this type, the characteristic type ap- 
A. Horizontal U loops in cases with ischemic heart disease

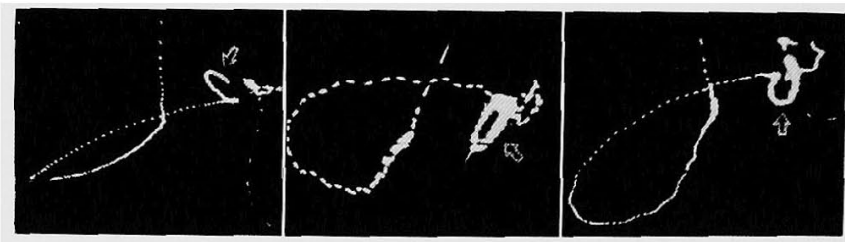

B. U loop in case with hyperpotassemia

i) Serum K, $4.5 \mathrm{mEq} / \mathrm{L}$.

ii) Serum K, $2.9 \mathrm{mEq} . / \mathrm{L}$.

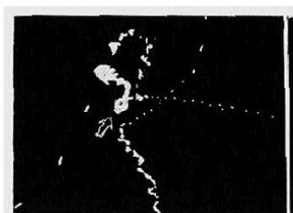

H.P.

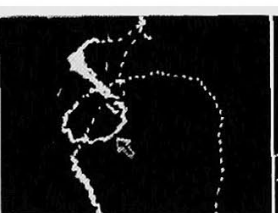

H.P.

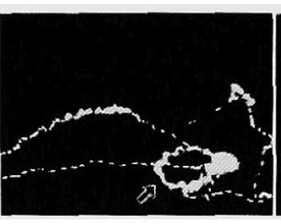

L.S.P.

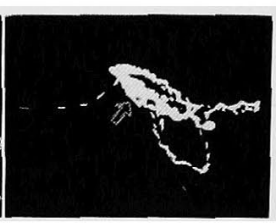

F.P.

C. Horizontal U loops in cases with myocardial infarction
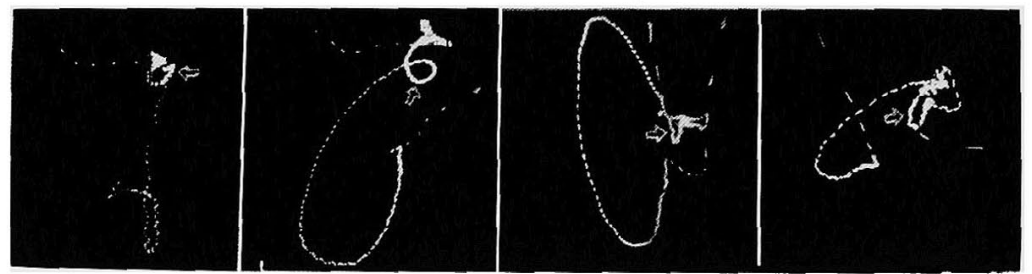

Fig. 3. Primary U loop change.

peared in this group. In this type, the middle portion of the $U$ loop was located rightanteriorly but the terminal portion remained leftanteriorly. Namely, the U loop of this type seemed to be pushed posteriorly from the location of the third type of hypertensive cases. In general, the U loop of the fourth and fifth types in this group also seemed to be pushed more posteriorly than in hypertensive cases.

Frontal Plane and Sagittal Plane: In this group, the U loop in these planes was not so markedly different from that in hypertensive cases.

Anterior and Anterolateral Infarction Group (Fig. $3 \mathrm{C}$ )

The $\mathrm{V}$ shape located rightposteriorly or elliptical shape in horizontal plane was seen in the cases with anterolateral infarction, but the horizontal $\mathrm{U}$ loop in anterior infarction was smaller that in anterolateral infarction.

\section{Discussion}

The significance of the $U$ wave in EGG has been reported by many authors. It is understood that the high $\mathrm{U}$ wave is caused by potassium de- 
ficiency. Recently, it has been reported that the negative $U$ wave appeared in some cases with hypertension and coronary sclerosis. However, the study of the U loop in VCG has not been reported except by Sano. Sano et al. have discussed the U loop in VGG with the Frank system. They described the U loop in cases of normal, left ventricular hypertrophy, right ventricular hypertrophy, right and left bundle branch block and infarction, but they did not mention the contour of abnormal U loop in detail. In their reports, the normal $\mathrm{U}$ loop resembled a small, slightly curved club and was inscribed almost in a direction of continuation of the terminal limb of the T loop; the $\mathrm{U}$ loop in left ventricular hypertrophy began with a marked band at the T-U junction and extended in various directions, also resembling a small curved club but sometimes much longer than the normal U loop.

The normal $U$ loop in this present study is not recognized as a club, but as a small loop or hook, when the enlargement is large enough to observe the $\mathrm{U}$ loop on an oscilloscope.

In cases with hypertension, the $\mathrm{U}$ loop is larger than normal and is easy to be recognized on the film or oscilloscope, and the contour and the direction of inscription are different from those of the normal $U$ loop.

In cases with the $T$ loop located rightanteriorly (Subgroup III), the horizontal $\mathrm{U}$ loop resembles a wedge ( $\mathrm{V}$ shape) and is inscribed clockwisely, and in many cases with the $\mathrm{T}$ loop located leftanteriorly or anteriorly (Subgroup I and II), the horizontal $U$ loop being $\gamma$ shape is located anteriorly or leftanteriorly. The contours of the $\mathrm{U}$ loop in the hypertensive group are shown in Fig. 2 A.

The first type of the $\mathrm{U}$ loop is the same as the normal $\mathrm{U}$ loop, and the second type is the $\mathrm{V}$ or $\gamma$ shape located leftanteriorly, being larger than the normal loop. The third type is $\gamma$ shape and located anteriorly. The fourth type is located rightanteriorly with $r$ shape. The last type shows $\mathrm{V}$ shape which is located rightanteriorly. Namely, in cases of hypertension, the magnitude of the $U$ loop is increased so that the $U$ loop becomes larger, and moves from leftanteriorly to rightanteriorly.

Finally the latter part of the U loop moves much further to the right than the former part and the $\mathrm{U}$ loop makes $\mathrm{V}$ shape. It does not always follow that shape in the second type is a more advanced type ( $V$ shape), but $V$ shape in the second type is not observed in the U loop of Subgroup II, and this V shape is completely different from $\mathrm{V}$ shape in the last type. As shown in Table I, the correlations between the numbers of each type of the U loop and Subgroup I-III indicate that these changes of the $U$ loop are parallel or preceeding to the dislocation of the $T$ loop and it is indicated that these $U$ loop changes are due to left ventricular hypertrophy. The changes of these types of the U loop 
Table I. Relation between the Contours of the U Loop and the Angle of the Maximum T Vector

\begin{tabular}{|c|c|c|c|c|}
\hline $\begin{array}{l}\text { Maximum } T \text { vector } \\
\text { (Subgroup) }\end{array}$ & $\begin{array}{l}<70^{\circ} \\
(\mathrm{I})\end{array}$ & $70^{\circ} \leq<110_{(1 \mathrm{I})}^{\circ}$ & $\begin{array}{c}110^{\circ} \leq 5 \\
\text { (III) }\end{array}$ & $\begin{array}{l}\text { Abnormal T } \\
\text { loop (IV) }\end{array}$ \\
\hline \multicolumn{5}{|l|}{ Hypertensive cases } \\
\hline \multicolumn{5}{|l|}{ U loop } \\
\hline normal type & 5 & & & \\
\hline first type & 5 & & & \\
\hline second type & 3 & 3 & & 4 \\
\hline third type & 1 & 6 & & 1 \\
\hline fourth type & 3 & 7 & & 4 \\
\hline fifth type & & 1 & 15 & 5 \\
\hline primary change & 2 & 4 & 1 & 18 \\
\hline \multicolumn{5}{|c|}{ Aortic insufficiency group } \\
\hline \multicolumn{5}{|l|}{ U loop } \\
\hline normal type & 1 & & & \\
\hline first type & 2 & & & \\
\hline second type & 2 & & & \\
\hline charact. type & 8 & 2 & & \\
\hline fourth type & & 3 & & \\
\hline fifth type & & 2 & 1 & \\
\hline large U loop & & 1 & 3 & \\
\hline
\end{tabular}

are called as the secondary $\mathrm{U}$ loop change.

In some cases of the hypertensive group, the U loop is round or located to the right or rightposteriorly. Such different type of the U loop are unlike to any $U$ loops observed in the 5 types, and these shapes are exceptional in Subgroup I-III.

In the abnormal $\mathrm{T}$ loop group (Subgroup IV), the $\mathrm{T}$ loop is round or elliptical and located in various directions. The horizontal $U$ loop of this group is located anteriorly, rightward or rightposteriorly, its contour being round or elliptical and large. These $U$ loops are very similar to the above mentioned exceptional types found in Subgroup I, II and III of the T loop of the hypertensive group and infarction group. This finding suggests that the round or elliptical U loop has no correlation to left ventricular hypertrophy, and we call these $\mathrm{U}$ loop changes as the primary $\mathrm{U}$ loop change.

It can be concluded that the horizontal $U$ loops in hypertensive cases are divided into 2 kinds of the $U$ loop. One is due to left ventricular hypertrophy and the other is due to myocardial injury which may be caused by coronary insufficiency or other factors. The contour of the $U$ loop in aortic insufficiency is similar to the $\mathrm{U}$ loop in hypertension. However, the charac- 
teristic type in aortic insufficiency corresponding to the third type in hypertension seems to be pushed more posteriorly than the third type. The T loop in aortic insufficiency is also different from that in hypertension. Namely, the $T$ loop located leftanteriorly or anteriorly in aortic insufficiency is round, the $\mathrm{T}$ loop in hypertension being long and narrow. The afferent limb of that round loop is dislocated to the right and that dislocation might be due to diastolic overloading of the left ventricle. The same phenomenon appears in the $\mathrm{U}$ loop and the first half is dislocated to the right and the middle portion seems to be pushed more posteriorly than that in hypertension. On the contrary, the terminal portion remains leftanteriorly as same as the efferent limb of the $T$ loop. In the anterior and anterolateral infarction group, the $U$ loop in horizontal plane is different from the contour of the secondary $U$ loop in hypertensive and aortic insufficiency groups, but is rather similar to the contour of the primary $\mathrm{U}$ loop. The horizontal $\mathrm{U}$ loop in hypopotassemia is large and round as shown in Fig. $3 \mathrm{~B}$ ii and becomes smaller when serum $\mathrm{K}$ level returns normal (Fig. $3 \mathrm{~B}$ i).

The development of the $U$ wave in EGG has been left unclear. It has been considered that $U$ wave resulted from an after potential similar to that of the nerve. ${ }^{3)}$ Another possibility is that the $U$ wave is caused by an elastic relaxation of the ventricular muscle. Maekawa ${ }^{4)}$ described that the abnormal high $U$ wave might be due to myocardial fatigue. Hoffman ${ }^{51}$ denied the theory of after potential, believing that the $U$ wave was the Purkinje fiber system $T$ wave. It seems impossible to determine the cause of the $U$ wave or the $U$ loop, but it is possible to differentiate the abnormal $U$ loop is due to ventricular hypertrophy or myocardial injury from their contours. And in most cases the displacement of the $\mathrm{U}$ loop is parallel or preceding to the $\mathrm{T}$ loop change, but in some cases, the change of their loops is not parallel to that of the T loop. So it can be said that the $U$ loop is affected in general by the same etiology which causes the change of the $T$ loop but the place which produces the U loop may be somewhat different from that of the $\mathrm{T}$ loop and the change of lateral wall of the left ventricle seems to affect contour of the horizontal $U$ loop much more than that of anterior wall.

\section{SUMMARY}

The authors observe the contour and location of the $U$ loop in normal, hypertension, aortic insufficiency and infarction.

(1) In normal cases, the horizontal U loop is located leftanteriorly with the small loop or $\mathrm{V}$ shape, and the left sagittal $\mathrm{U}$ loop is located anteroinferiorly with small loop. The U loop in frontal plane is located leftinferiorly with hook 
or $\gamma$ type.

(2) In hypertension, the U loop in horizontal plane is dislocated from leftanteriorly to rightanteriorly. The contour of the $\mathrm{U}$ loop is $\gamma$ or $\mathrm{V}$ shape and in cases with the $\mathrm{T}$ loop located rightanteriorly with clockwise inscription, the $\mathrm{U}$ loop is $\mathrm{V}$ shape with clockwise inscription. The authors call those $\mathrm{U}$ loop as the secondary $U$ loop change.

(3) In aortic insufficiency, the $\mathrm{U}$ loop change in horizontal plane is similar to that in hypertension, but, in some cases the middle portion of the horizontal $\mathrm{U}$ loop is located rightanteriorly, the terminal portion remaining leftanteriorly. This characteristic type corresponds to the third type in hypertension and might be due to diastolic overloading.

(4) In some cases with hypertension and many cases with anterior or anterolateral infarction, the horizontal $\mathrm{U}$ loop is dislocated to the right or rightposteriorly or is shaped with round or ellipse. These changes of the U loop are called as the primary $U$ loop change.

(5) The genesis of the $U$ loop is discussed with those results.

\section{REFERENCES}

1. Takayasu, M.: Coronary disease and electrocardiographic findings, Proceeding of the 16th General Assembly of the Japan Medical Congress, 1963.

2. Sano, T., Tsuchihashi, H., Takigawa, S., and Shimamoto, T.: Am. Heart J. 61: 802, 1961,

3. Lepcschkin, E.; Modern Electrocardiography Vol. I., Williams \& Wilkins Co., Baltimore, 1951.

4. Hayase, S.: Sogo Rinshyo 8: 20431959 (in Japanese).

5. Hoffman, B. F. and Cranefeld, P. F.: Electrophysiology of the Heart, McGraw-Hill Book Co., New York, 1960. 